\title{
Open Surgical Management of Abdominal Aortic Aneurysm at a Community Based University Hospital in Nepal Bhandari $N,{ }^{1}$ Karmacharya RM, ${ }^{1}$ Devbhandari $M,{ }^{1}$ Shrestha $B,{ }^{1}$ Singh $A K,{ }^{1}$ Ranjit $S,{ }^{2}$ Shrestha $K R^{3}$
}

\author{
${ }^{1}$ Department of Surgery \\ ${ }^{2}$ Department of Anesthesia, \\ Dhulikhel Hospital, Kathmandu University Hospital \\ Dhulikhel, Kavre, Nepal. \\ ${ }^{3}$ Department of Vascular Surgery \\ Manmohan Cardiothoracic Vascular and Transplant \\ Center \\ Maharajgunj, Kathmandu, Nepal.
}

\section{Corresponding Author}

Niroj Bhandari

Department of Surgery

Dhulikhel Hospital, Kathmandu University Hospital

Dhulikhel, Kavre, Nepal.

\section{Citation}

Bhandari N, Karmacharya RM, Devbhandari M, Shrestha B, Singh AK, Ranjit S, Shrestha KR. Open Surgical Management of Abdominal Aortic Aneurysm at a Community Based University Hospital in Nepal. Kathmandu Univ Med J. 2020;69(1):96-8.

\begin{abstract}
Aortic aneurysms are abnormal dilatation of aorta. The risk factors include male sex, age $>65$, smoking, coronary artery disease and hypertension. Here we report a case of infra-renal abdominal aortic aneurysm (AAA) of diameter $6 \mathrm{~cm}$. The patient sucessfully underwent aorto-biiliac bypass surgery using Dacron $Y$ graft. During abdominal aortic aneurysm surgery anesthetic challenge is also of paramount importance and should be considered.
\end{abstract}

\section{KEY WORDS}

Abdominal aortic aneurysm, Aorto-biiliac bypass, Dacron graft, Vascular surgery

\section{INTRODUCTION}

Aortic aneurysm is defined as abnormal dilatation of aorta with more than $50 \%$ increase in diameter or more than $30 \mathrm{~mm}$ on imaging most commonly affecting infrarenal region. ${ }^{1}$ Abdominal aortic aneurysm is one of its variant which is characterized by pain, pulsatile mass in abdomen, sometimes shock due to rupture and rarely can have features of thrombosis. ${ }^{2,3,4}$ Smoking is the most important modifiable risk factor followed by coronary artery disease, hypertension. ${ }^{5}$ In regards to non modifiable risk factors, male sex and age older than 65 are important risk factors. ${ }^{2,3}$

\section{CASE REPORT}

A 72 years male from Kapan, Kathmandu presented to the surgical out patient department of Dhulikhel Hospital, Kathmandu University Hospital, Dhulikhel with history of pain in upper central abdomen for one month which increased on exhaustion. On examination pulsatile non tender mass of size $7 \times 5 \mathrm{~cm}$ was palpated in central abdomen. He is also known case of hypertension and is controlled under medication. The abdominal sonogram showed abdominal aortic aneurysm of size $5 \times 5 \mathrm{~cm}$. Contrast Enhanced CT angiogram confirmed the finding along with extent of $7 \mathrm{~cm}$ distal from right renal artery to bilateral common iliac artery. In view of size of aneurysm and symptomatic status bypass of the aneurysmal segment was planned. For surgical correction abdominal aorta proximal to the aneurysm was planned to be bypassed to bilateral external iliac arteries using Dacron Y graft. Details are shwon in figure 1, 2 and 3. 


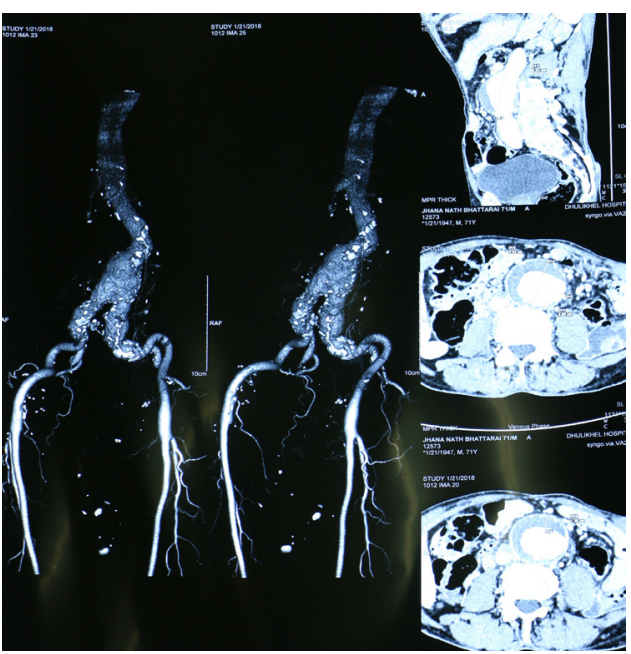

Figure 1. 3D reconstruction of the lesion.

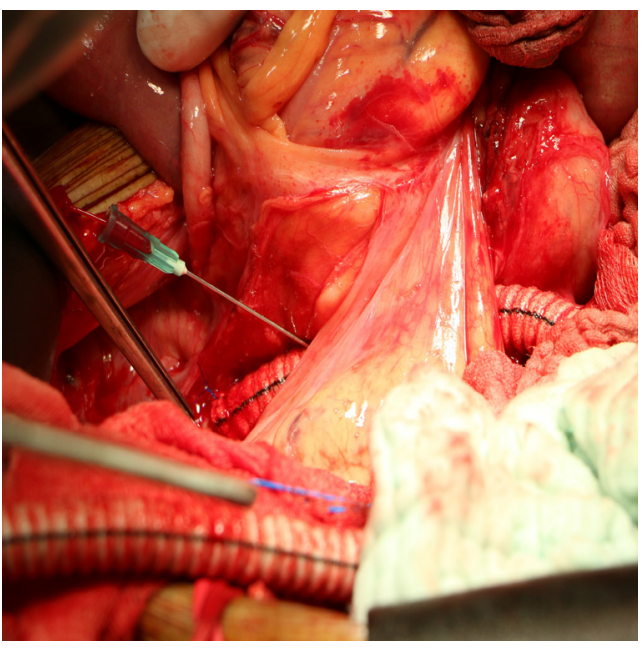

Figure 2. Distal anastomosis of $Y$ graft to right external iliac artery

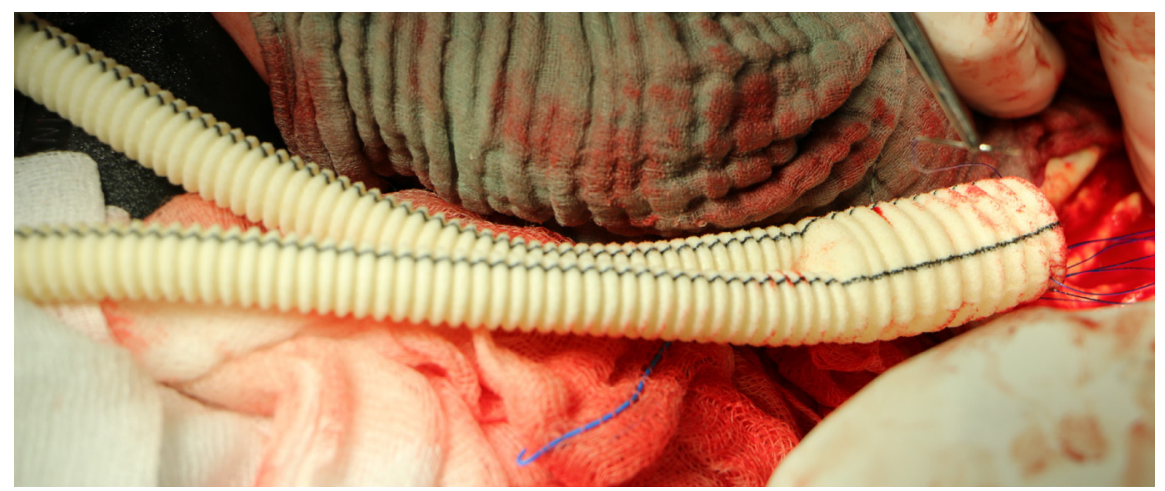

Figure 3. Proximal end of $Y$ graft during reconstruction

Intraoperative findings were similar to the CT findings. After placing clamps in proximal and distal part aneurysmal sac was opened to find atheroma. Proximal end of $Y$ graft was connected to abdominal aorta by parachuting technique (end to end anastomosis) using 5 "0" prolene. Following de-airing of distal limbs of $Y$ graft, the ends were tunneled below mesentery to be anastomosed to bilateral external iliac artery. Right limb of graft was further anastomosed to right internal iliac artery using short tubular graft. Post procedure good pulse was appreciated in bilateral femoral arteries. Patient recovered uneventfully with two days of ICU stay and seven days of hospital stay. In follow up of a year the bypass is patent with adequate flow and no evidence of distal ischemia.

\section{DISCUSSION}

Guidelines from American College of Cardiology/American Heart Association 2005 has given the recommendation for surgical repair to be done in case the AAAs are $5.5 \mathrm{~cm}$ in diameter or greater for asymptomatic patients while increase in diameter by $0.5 \mathrm{~cm}$ or greater in six months is indicative of surgery in cases of symptomatic patients. ${ }^{6}$ AAAs are classified based on anatomic site and extent into Suprarenal, Pararenal, Juxtarenal and Infrarenal types. ${ }^{7}$

During surgery for abdominal aortic aneurysm, anesthetic challenge is also of paramount importance owing to the risks during aortic clamping and de-clamping. Clamp application causes sudden increase in afterload and after clamp release there is sudden decrease of peripheral vascular resistance by $70-80 \%$ causing hypotension. ${ }^{8}$ Similarly ischemia-reperfusion cascade also need to be taken into consideration during surgery. ${ }^{8}$

In recent time development in minimal invasive endovascular treatment such as Endovascular Aortic Repair which cause less morbidity and mortality but such modalities are costly and technologically more demanding. ${ }^{9}$

Surgery for AAA is complex, skill and resource demanding procedure hence successful management of such case at tertiary level hospital of periphery shows improvement in vascular surgery status of Nepal. Timely consultation and appropriate treatment with multi-disciplinary approach is essential for AAA manangement. 


\section{REFERENCES}

1. Tiwari KK, Bevilacqua S, Salvati A, E Varone, M Solinas, M Glauber. Ascending Aortic Aneurysm, Wandering towards Old Problem with New Insight. Journal of Universal College of Medical Sciences. 2014; 2: 49-59.

2. Golledge J. Abdominal aortic aneurysm: update on pathogenesis and medical treatments. Nat Rev Cardiol. 2018; 1.

3. Keisler B, Carter C. Abdominal Aortic Aneurysm. AFP. 2015; 91: 53843.

4. Seidenberg B, Stern J, Hurwitt ES. Thrombotic Occlusion of Abdominal Aortic Aneurysm Following Distal Embolization. Circulation 1962; 25: 995-6.

5. Lederle FA. The Aneurysm Detection and Management Study Screening Program: Validation Cohort and Final Results. Arch Intern Med 2000; 160: 1425.

6. Aggarwal S, Qamar A, Sharma V, Sharma A. Abdominal aortic aneurysm: A comprehensive review. Exp Clin Cardiol. 2011; 16: 11-5.

7. Chaikof EL, Blankensteijn JD, Harris PL, White GH, Zarins CK, Bernhard VM, et al. Reporting standards for endovascular aortic aneurysm repair. Journal of vascular surgery. 2002 May 1;35(5):1048-60.

8. Al-Hashimi M, Thompson J. Anaesthesia for elective open abdominal aortic aneurysm repair. Continuing Education in Anaesthesia Critical Care and Pain. 2013; 13: 208-12.

9. Chaikof EL, Dalman RL, Eskandari MK, Jackson BM, Lee WA, Mansour MA, et al. The Society for Vascular Surgery practice guidelines on the care of patients with an abdominal aortic aneurysm. J Vasc Surg. 2018; 67: 2-77.e2. 